\title{
Adverse events in health care: a literature review
}

ANNE-MARIE BRADY BSN, MS PG Dip. Clinical Health Sciences Ed RGN, RNT, RICHARD REDMOND MEd, PhD, RPN, RNID, RNT, FFNMRCSI, ELIZABETH CURTIS PhD, MEd, DMS, Dip. Research Methods, RGN, ONC, SANDRA FLEMING MSc Cert. Ed (FE), RNID, RPN, RGN, RCT, RNT, PAUL KEENAN MSc, BSc, PGCE, Dip. HE NP, Dip. RS, RNID, ANNEMARIE MALONE mbA, BNS, RNT, RCN, RGN, RM and FINTAN SHEERIN PhD, BNS PG Dip. Ed, RNID, RGN, RNT Lecturer, School of Nursing \& Midwifery, Trinity College Dublin, Dublin, Ireland

Correspondence

Anne-Marie Brady

School of Nursing \& Midwifery

Trinity College Dublin

24 D'Olier St

Dublin 2

Ireland

E-mail: anne-marie.brady@tcd.ie
BRADY A.-M., REDMOND R., CURTIS E., FLEMING S., KEENAN P., MALONE A.-M. \& SHEERIN F. (2009) Journal of Nursing Management 17, 155-164

Adverse events in health care: a literature review

Aim This paper aims to develop understanding of the nature, costs and strategies to reduce or prevent a range of adverse events experienced by people within the healthcare system.

Background Care interventions are not always based on safe practice and adverse events can and do occur that cause or place at risk patients lives and well-being. The nature of adverse events is diverse and can be attributed to a multitude of individual and system contributory factors and causes.

Evaluation A review of the literature was undertaken in 2006 and 2007 using the following databases: Pubmed, CINAHL, Biomed Ovid, Synergy and the British Nursing Index. This paper evaluates the literature that pertains to adverse events and seeks understanding of this complex issue.

Key issues Published statistics confirm that globally, professional errors in clinical practice and care delivery occur at an unacceptably high level and result in considerable human and financial consequences.

Conclusion Reaching understanding of the multiple factors that contribute to unsafe clinical practice situations requires a cultural shift in organizations.

Implication for Nursing Management Reasons for adverse events are complex and require healthcare managers to evaluate the system issues which impact on the delivery and organization of care.

Keywords: adverse events, medical error, patient safety, quality

Accepted for publication: 5 March 2008

\section{Introduction}

In general, when an individual accesses the healthcare system, they will have an expectation that the care and treatment they receive will be delivered by competent professionals in a safe environment and without unintended harm to their well-being. However, this is often not the case as many patients experience harm while a patient. The safety of the patient is now recognized DOI: 10.1111/j.1365-2834.2008.00887.x

(c) 2008 The Authors. Journal compilation @ 2008 Blackwell Publishing Ltd globally as a healthcare priority [World Health Organisation (WHO) 2006]. Reports that the number of patient deaths from adverse events in healthcare could be higher than previously thought have led to renewed interest in patient safety (Classen et al. 1997, Fine 2004). Reports prepared by the Institute of Medicine (1999) and Brennan et al. (1991) in the USA served as the catalyst for increased awareness of this problem and the need to take action as a matter of urgency. 
Consequently, hospital administrators and health service managers are focusing attention on reducing the prevalence of adverse events and ways to improve patient safety. This paper has a fourfold aim. First, it discusses the concept and nature of adverse events along with their incidence and prevalence in health care. Second, it reviews literature on the cost, financial and human, of such adverse events. Third, it discusses strategies useful for reducing the incidence of adverse events. Fourth, it will help sensitize the reader to the need for preventing adverse events.

\section{Nature of adverse events}

Moullin (2002) describes harm caused to the individual while a patient as an adverse event (AE) of health care. Moullin sees adverse events which harm, threaten or compromise the safety of a patient as stemming from two main sources: direct interventions from professionals as they carry out their treatment or care for the patient and, indirect exposure of the patient to unnecessary and potentially harmful risks from the healthcare environment while a patient. Adverse, according to Collins Dictionary (1999), refers to something that is unfavourable to one's interests while an adverse event refers to a change or state of affairs as a result of that something. Applied to healthcare the concept is complex. Hiatt (1989) have explained an adverse event as unintended injuries as a result of medical management (not the underlying disease) and which result in measurable disability. Adverse event studies according to Howard (2003) measure events that have resulted in injury whereas studies of adverse events from prescribing medication can also include near misses as well as injury to patients. The International Conference on Harmonization defined an adverse event as any untoward medical occurrence in a patient or investigational participant to whom a medical product has been administered, including occurrences that are not necessarily caused by or related to that product $(\mathrm{ICH}$ 1996). In a detailed review of adverse drug reactions, Edwards and Aronson (2000) make a distinction between the terms adverse effect and adverse event. They see an adverse effect as an outcome attributed to some adverse action of a drug whereas an adverse event is an adverse outcome occurring while a patient is taking a drug but which is not necessarily attributable to it. Edwards and Aronson (2000: 1255) define an adverse drug reaction as:

An appreciably harmful or unpleasant reaction, resulting from an intervention related to the use of a medicinal product, which predicts hazard from future administration and warrants prevention or specific treatment, or alteration of the dosage regimen or withdrawal of the product.

As a result of its complexity, many distinct disciplines of study advance their own theories to explain and provide an understanding of the concept of adverse events. Four particular perspectives that make a contribution to understanding adverse events include medical, sociological, psychological and continuous quality movement. In his discussion on the medical perspective, Esmail (2006) argues the fallible nature of medicine which is inherently unsafe, where doctors' thinking is embedded in medical culture and the norm is for members of the profession to come together to maintain the status quo and the norm of non-criticism. Esmail (2006) cites an early paper by Gorovitz and MacIntyre (1976) who suggested three reasons for medical fallibility. First was ignorance because science provides only limited understanding of how things happen. Second was ineptitude where knowledge is available but where doctors fail to apply it in the correct manner. Third was termed necessary fallibility because there is some knowledge that science and technology will never provide.

The sociological perspective as advanced by West (2006) puts forward the claim that most of the adverse events occurring in hospital are system failures rather than individual failures as many of the errors are rooted in the features of the organization. Complex organizations such as hospitals are vulnerable to breakdowns in communication and coordination, because of the tendency of individuals to relate to others within their own practice discipline barriers are created to working in groups. The Department of Health (DoH) (1999) in the UK acknowledge the need to move the culture of healthcare organizations away from blaming individuals towards an analysis of systemic sources of error. West sees sociology as providing a useful theoretical framework for understanding and studying adverse events as it shifts attention away from the individual towards the group level of analysis.

The psychological perspective on understanding adverse events is centred on theories that explain behaviour, motivation and organizational learning. Parker and Lawton (2006) see the psychological perspective going beyond the description to explanation because it includes a consideration of the cognitive and motivational underpinnings of error. Having a personcentred approach to dealing with adverse events focuses attention and blame on the individual involved, and 
once the individual is found the investigation is closed with the loss of organizational learning. In addition, Parker and Lawton point out that in an organizational culture where individuals are blamed for adverse events there is a likelihood that such events (especially near misses) will go unreported whenever possible. With the systems approach, to be able to understand the roots of individual error it is necessary to consider the physical, social and organizational environments in which individuals work.

Another perspective for understanding the occurrence of adverse events is through quality improvement. Moullin (2002) has emphasized the part quality improvement programmes play in identifying and helping to reduce adverse events. Some quality programmes may be specific to professionals only and while these are important for standards and clinical audits, they cannot take account of the larger hospitality elements of a hospital where adverse events can and do occur (Parsley \& Corrigan 1999). Many healthcare organizations have quality programmes that are set at an organizational-wide level such as continuous quality improvement or total quality management. Such programmes have a system-wide approach and many are linked to accreditation. Hackman and Wageman (1995) see such programmes as focusing on work processes, management systems, controlling for variability, customer requirements and cross-functional teams for problem solving. As these activities focus on people's behaviour, quality programmes can make an important contribution to avoiding adverse events by identifying unsafe practices.

A number of contributory factors have been cited including fragmentation of health services as outlined in the 'To Err is Human Report' (Institute of Medicine
1999). Errors are seldom caused by personal failing, inadequacies or carelessness (Leape 2000) nor do they occur because of the reckless actions of individuals (Institute of Medicine 1999). They are more commonly a result of defective approaches to work design and conditions of healthcare work leading to conditions where mistakes can occur (Institute of Medicine 1999, Leape 2000).

\section{Frequency of adverse events}

Screening for adverse events is an essential part of the quality-improvement process (Karson \& Bates 1999). There is growing interest nationally and internationally in developing voluntary and mandatory standardized reporting systems to record adverse events to provide baseline information [Institute of Medicine 1999, DoH (2000), Health Care Commission 2006]. It is estimated that between 44000 and 98000 deaths in US hospitals each year can be attributed to preventable medical error (Institute of Medicine 1999). In the UK, it is estimated that adverse events occur in $10 \%$ of all admissions or at a rate of 850000 patients per year (DoH 2000). The National Patient Safety Agency (NPSA) identified the types and frequency of patient safety incidents $(n=67344)$ in acute care settings in the NHS as illustrated in Table 1 (NPSA 2005). Common medical errors that can occur during the provision of healthcare services include adverse drug events, wrong-site surgery, restraints injuries, falls and pressure sores with significant physical, psychological, organizational and societal consequences (Considine \& Botti 2004).

The first and most significant study of the rates of adverse events was undertaken by the Harvard School of Public Health in 1991 [Harvard Medical Practice
Table 1

Reported patient safety incidents in acute care settings

\begin{tabular}{lr}
\hline Incident type & $n(\%)$ \\
\hline Patient accident (slips, trips and falls) & $30363(44.6)$ \\
Treatment, procedure & $6632(9.8)$ \\
Medication & $5797(8.6)$ \\
Infrastructure (including staffing, facilities, environment) & $4526(6.7)$ \\
Access, admission, transfer and discharge & $3863(5.7)$ \\
Documentation (including records, identification) & $3746(5.6)$ \\
Clinical assessment (including diagnosis, scans, tests) & $3065(4.6)$ \\
Consent, communication, confidentiality & $2894(4.3)$ \\
Medical device/equipment & $2709(4.0)$ \\
Implementation of care and ongoing monitoring review & $1352(2.0)$ \\
Infection control & $624(0.9)$ \\
Disruptive aggressive behaviour & $440(0.7)$ \\
Patient abuse (by staff/third party) & $129(0.2)$ \\
Other & $1504(2.2)$ \\
Total & $67344(100)$
\end{tabular}

Source: National Patient Safety Agency (2005: 47). 
Study (HMPS)] and it provided the first concrete evidence on the frequency and type of medical errors (Brennan et al. 1991, Leape et al. 1991). The researchers reviewed 30121 randomly selected medical records from 51 randomly selected acute care New York hospitals in 1984 (Brennan et al. 1991). Two physician reviewers independently identified, evaluated and categorized the nature of the adverse events (Leape et al. 1991). The most common type of adverse events found were drug complications $19 \%$, wound infections $14 \%$ and technical complications $13 \%$. The most significant finding of the study was the rate of adverse events calculated at $3.7 \%$ [95\% confidence interval (CI) 3.2-4.2] of all hospitalizations. The researchers estimated that $27.6 \%$ (CI: 22.5-32.6) of all adverse events were as a result of negligence. Other findings provide insight into the proportion of adverse events caused by negligence, including diagnostic mishap (75\%) and non-invasive therapeutic mishaps or errors of admission $(77 \%)$. Errors of admission have been described as failure to anticipate or act upon information at the diagnosis, treatment or therapeutic monitoring phases of the patient care experience (Leape et al. 1991). In this study $13.6 \%$ of adverse events led to death.

This study was followed in 1992 by a study that used similar methods to estimate the incidence and types of adverse events in Utah and Colorado (Thomas et al. 2000a). Trained nurse reviewers retrospectively screened 15000 medical charts using 18 agreed criteria indicative of adverse events. If any criteria were present, the chart was subsequently analysed by a physician to identify and categorize the type of adverse event. The rates of iatrogenic injury were found to be similar to the Harvard study in 1984 with an adverse events rate of $2.9 \%$ (SD $0.2 \%$ ). In Utah, of those patient admissions with adverse events, negligence was found to be the cause in $32.6 \%$ (SD $4 \%$ ) of cases and in Colarodo $27.4 \%$ (SD $2.4 \%$ ). Surgery was associated with $44.9 \%$ of all adverse events with adverse drug events found to be the leading cause of non-operative adverse events $(19.3 \%)$. Death occurred in $6.6 \%$ of all adverse events. These two US studies used the HMPS protocol and have been criticized particularly in relation to the reported death rates, as the studies did not provide baseline information about the risk of death in these or in a control group and the research design was not capable of establishing causal relationships (McDonald et al. 2000). However, Leape (2000) reported that this criticism is misguided and may be as a result of the physicians' need to absolve their burden of guilt.

The Australian Quality of Health Care (QAHCS) study also used the two-stage retrospective chart review of 14179 patients records in both public and private hospitals $(n=28)$ and reported a preventable adverse event rate of $16.6 \%$ in all hospital admissions and that $50.3 \%$ of those were associated with surgery (Wilson et al. 1995). A follow-up analysis exclusively examined the adverse event rate for the 5432 surgical patients in the 28 randomly selected hospitals (Kable et al. 2002) and found the AE rate for surgical admissions to be $21.9 \%$. The Australian results represent a much greater rate than the US studies and this disparity has subsequently been analysed by a team of researchers from both the US and Australia in an effort to account for the considerably different rates recorded in studies using similar protocols (Thomas et al. 2000b). While both studies used a similar two-stage chart review process to detect adverse events, five methodological differences occurred in the Australian study which, when adjusted reduced the rate to $10.6 \%$. While this remains much larger than the prevalence in the USA, the authors do offer a number of possible explanations that may explain why Australian rates remain much higher than their counterparts in the USA. Different research goals, use of specialist reviewers, differences in the nature of medical records, reviewer behaviour and differences in standards of care were some of the reasons offered to explain the differences in results.

The criteria established in HMPS and QAHCS studies were used in a retrospective review of 1014 medical and nursing records in the UK (Vincent et al. 2001). In this study, conducted at two London teaching hospitals, the researchers sought to establish the feasibility of detecting adverse events through record review in British Hospitals. In 1999/2000, 500 and 514 records were randomly selected from the two sites respectively. The review process was initiated by nurses who screened charts for the 18 predefined criteria. Of those, 405 were reviewed by physicians and assessed for impact of each adverse event. These included consideration of impact on patients, additional bed days, possible causes and types of adverse events. The findings were that 110 $(10.8 \%)$ out of 1014 patients assessed had an adverse event and such AEs contributed to death in nine $(8 \%)$ patients. The researchers concluded that a full national study is justified in the UK and estimated that the cost of such a study would be equivalent to the amount lost by the NHS in any 8 -hour period, through preventable AEs (Vincent et al. 2001).

Chart reviews were conducted by a team of Danish researchers on 1097 admissions to 17 acute hospitals (Schioler et al. 2001). The prevalence of admissions with adverse events was found to be $9 \%$, similar to those results previously found in Australia, UK and 
USA. A two-stage retrospective review of 6579 medical records in New Zealand in 1998 (Davis et al. 2002) established that the proportion of adverse events was $12.9 \%$. Nearly one-fifth of those were found to have occurred before admission to a public hospital. A similar Canadian study in 2000, $(n=1527)$ reported that the rate of adverse events in five Canadian provinces was $7.5 \%$ (Baker et al. 2004). Table 2 provides an overview of the major studies into the rate of adverse events conducted. There are a variety of retrospective and prospective screening techniques including incident reporting, clinical scenario analysis, real-time reviews and generic record screens that may be used to detect adverse events in health care, with varying limitations, degrees of sensitivity or accuracy and cost implications (Karson \& Bates 1999). Refinement of techniques with greater sophistication is emerging with increasing computerization offering the opportunity for screening to be commonplace using methods that readily enable comparison across health care (Karson \& Bates 1999).

\section{Financial cost of adverse events}

Adverse events have significant cost implications. Financial losses accrue for many reasons including increased length of hospitalization for clients and resulting absence from the workforce, costs in the management of risk for individual entities and healthcare systems, the administration of patient safety agencies and the cost of medical litigation. The overall costs are also influenced by whether any resulting disability or harm to clients is short or moderate term, permanent or results in death (see Table 3).

There are also the non-financial costs including those of increased pain, suffering and anxiety for patients and significant others related to the experience of an adverse event, loss of confidence and trust in the healthcare system and increased patient dissatisfaction. James (2004) notes that the costs of adverse events in health care are funded through higher insurance premiums and higher taxes.

Specific areas that are highlighted in the published literature are costs associated with an increased length of stay, costs associated with medication errors, overall costs to the healthcare system and total cost to society as a whole. Increased length of stay related to adverse events in the research varies at 1.74 days for medication-related adverse events (Classen et al. 1997), to 8.1 days (Kalish et al. 1995) and 8.5 days (Vincent et al. 2001) for other types of adverse events. Vincent et al. (2001) note an $8 \%$ increased length of stay for patients who had experienced adverse events with associated costs to the Australian healthcare system of $\$ 4.7$ billion in 1 year alone. The Report of an Expert Group on learning on adverse events in the NHS (DoH 2000), state that the cost of increased length of stay alone was at that point $£ 2$ billion per annum to the NHS. Conversely, Vincent et al. (2001) estimate that the costs of adverse events in the NHS at the lower figure of $£ 1$ billion per year for increased length of stay.

The National Audit Office (2005) estimated the costs of settled litigation claims to the NHS at $£ 423$ million for the year 2003-04, with the expected costs of outstanding negligence claims for the same year at in excess of $£ 2$ billion. Other studies have delineated the cost of adverse events to individuals and society as a whole. One example is a study by Johnson et al. (1992) who endeavoured to ascertain the total costs of adverse events in New York State. They estimated the cost of adverse events at $\$ 21.4$ billion, with $\$ 2.6$ billion related to lost earnings, $\$ 3.4$ billion in lost household production and \$15.4 in medical expenses; $\$ 3.8$ billion was related to the injuries received. Thomas et al. (1999) recognized that previous published research was limited in that few studies estimated the total costs of adverse events to society as a whole. Therefore they endeavoured to estimate the national cost of adverse events in the state of Utah and Colorado in the USA. They estimated that the total costs of adverse events were $£ 348081000$ and that the costs of preventable adverse events were \$159245000.

The Joint Commission on Accreditation of Healthcare Organisations (JCAHO 2005) notes the costs associated with litigation as a result of adverse events and correspondingly recommends reform of the tort system to contain the escalating costs associated with litigation. Roberts and Hoch (2007) examined the cost of malpractice litigation on area medical costs for Medicare Part B patients in Mississippi, and estimated that the additional costs in counties that were plaintiff friendly were $25 \%$ higher. These researchers attribute this to the practice of defensive medicine. One example of the costs of a patient safety agency is the Canadian system, which in the initial stages was budgeted at $\$ 50$ million over a 5 -year period (Baker et al. 2004).

Extrapolating the total costs of adverse events in any healthcare system is problematic. The published research has been carried out internationally, over decades and utilizes different research methodologies to report on different aspects of costs associated with adverse events. The consistencies in these findings indicate that the costs of adverse events present a 


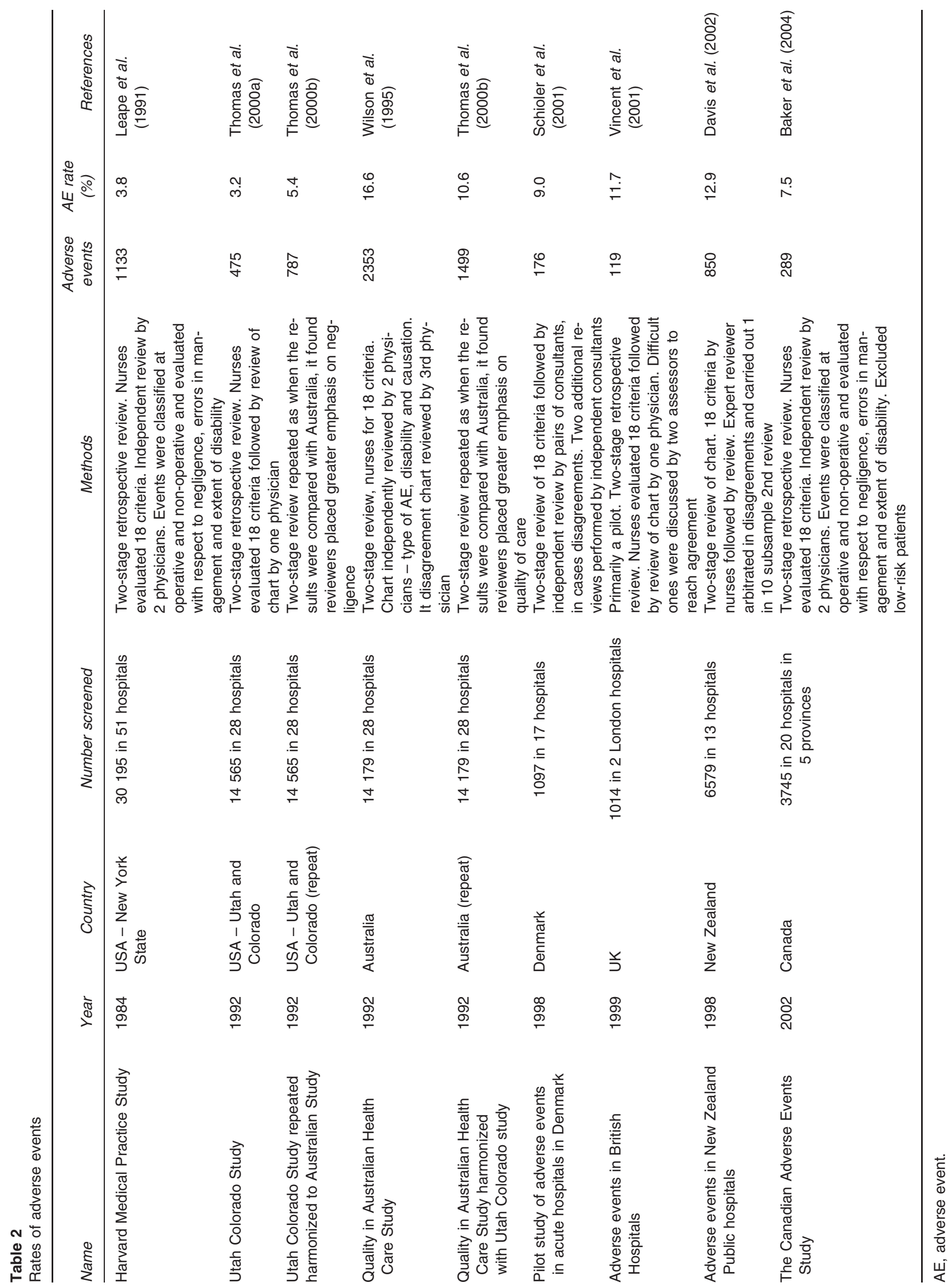


Table 3

Disability/harm rates associated with adverse events

\begin{tabular}{lcc}
\hline Type of disability & Rate & References \\
\hline Short term & $66 \%$ & Vincent et al. (2001), Brennan et al. (1991), \\
& & Leape et al. (1991) \\
Minor or minimal harm & $25 \%$ & National Patient Safety Agency (2006) \\
Moderate & $34 \%$ & Vincent et al. (2001) \\
Moderate harm & $5 \%$ & National Patient Safety Agency (2006) \\
Permanent & $6 \%$ & Vincent et al. (2001) \\
Permanent (severe) & $0.9 \%$ & National Patient Safety Agency (2006) \\
Death & $8 \%$ & Vincent et al. (2001) \\
& $0.4 \%$ & National Patient Safety Agency (2006)
\end{tabular}

significant economic burden to individuals, healthcare systems and society as a whole. However, given the limitations of the current published literature, the variations in healthcare systems globally, the total costs within any jurisdiction can only be estimated.

\section{Strategies to reduce adverse events}

Common challenges for patient safety have been identified globally. The World Alliance for Patient Safety (WAPS) was launched in October 2004 to encourage member states 'to pay the closest possible attention to the problem of patient safety and to establish and strengthen science-based systems necessary for improving patient safety and the quality of health care' (WHO 2006: 5). WAPS recognizes that errors exist in all WHO member states and no country can state that they have fully solved the problem of patient safety. There is a need to address safety problems whose causes and solutions are already well known. As detection systems are universally described as primitive there is a need for timely identification of new issues and their solutions. The dominant culture in health care is seen as one of blame and retribution most often directed at the individual rather than the organization's systems (Health Care Commission 2006). Many adverse events are not being identified and when they are, they are not always being reported (WHO 2006). This both detracts from addressing the problem at systems level and hinders opportunities for organizational learning. As a result, adverse events increase and safety is prevented from flourishing. The Organization for Economic Cooperation and Development (OECD) initiated the Health Care Quality Indicator (HCQI) Project in 2001 and the key purpose is to develop a set of indicators that could be used to raise questions for further investigation concerning quality of health care across countries (Kelley \& Hurst 2006). Quality indicators relate to the technical quality of medical care or health outcomes and could be said to represent the 'value for money' component in health care (OECD 2007). When completed the indicators will be designed to be scientifically sound, relevant at clinical and policy level and universally adopted across all member states so that areas of concern for patient safety can be identified and addressed (Kelley \& Hurst 2006).

Within Ireland and the UK, health services have undergone significant restructuring and reform in recent years with an increased emphasis on patient safety [DoH 2000, Department of Health and Children (DHC) 2001]. In Ireland, the general principles enshrined within the health strategy 'Quality and Fairness 2001' emphasize the importance of equity, accountability, quality and person-centeredness all of which drive policy initiatives in relation to patient care and safety (DHC 2001). In the UK, the National Patient Safety Agency outlined seven steps that encompass both the creation of a safety culture, and the systems and processes that underpin it (NPSA 2004). The guide is not prescriptive and NHS organizations are able to prioritize the actions according to local need. The seven steps to improve NHS organizations' patient safety may be summarized as follows:

1 Build a safety culture by encouraging staff to report when things go wrong (look at 'what' has gone wrong rather than 'who').

2 Lead and support staff by establishing a clear patient safety focus throughout the organization.

3 Integrate risk management activity by developing systems and processes to manage your risks and identify and assess things that could go wrong.

4 Promote reporting by ensuring that staff can report both locally and nationally when things go wrong.

5 Involve and communicate with patients and the public by developing ways to communicate with them.

6 Learn and share safety lessons by encouraging staff to analyse the root cause of incidents.

7 Implement solutions to prevent harm by learning lessons and making changes to practices, processes and systems (NPSA 2007). 


\section{Organization strategies}

No adverse event should ever occur if the knowledge exists to prevent it from happening. To have most effect, knowledge must be translated into solutions that can be applied to practice to make unsafe situations safe (WHO 2006). Designing effective solutions to adverse events is an organizational wide responsibility, which must include strategies to identify, detect and communicate potential adverse events to all concerned. In addition, organizations are required to ensure that all staff are competent, conscientious and safety conscious (WHO 2006). To enhance patient safety, organizations must create partnerships with all stakeholders (Doolan $\&$ Bates 2002). Stakeholders within any healthcare environment can include all healthcare professionals, administrators, support personnel, patients and members of the general public. The intention here is for stakeholders to work together to generate ideas that can be transformed into workable solutions regarding patient safety.

Open discussion about adverse events must be encouraged within organizations. This can be achieved through quality-improvement initiatives at all levels of the organization. The aim is to develop a climate where all employees feel at ease discussing issues such as adverse incidents. Effective systems for reporting errors without attachment of blame to an individual must be designed (Leape 1994). Adverse events will continue to rise unless a systematic system for reporting such events is implemented. An effective reporting system must be confidential, promote the reporting of adverse events, be non-partisan and ensure no reprisal against those reporting the events.

A cultural shift is required within organizations in order to improve patient safety (Ruchlin et al. 2004). Culture refers to beliefs and values shared by employees in the organization. Creating a safety culture requires not only making patient safety a top priority within the organization but also commitment from all stakeholders to address patient safety issues. Rather than adopting the standard approach of naming, blaming and shaming the individual when an error or adverse event occurs, a safety culture encourages the reporting of such events in a non-punitive manner. Eliminating or reducing adverse events requires continuing education and training. In addition, all staff require training on adverse events reduction techniques (Becher \& Chassin 2001). Such training might involve organized sessions on risk management directed toward identifying, evaluating and taking action against potential risks. Training of this nature is not only beneficial for improving patient safety but will also contribute to the reduction of healthcare litigation.

Statistical analysis on adverse events will be beneficial to organizations wishing to reduce such events (Becher \& Chassin 2001). Data may be collected on adverse events but without effective analysis it is meaningless. Quantitative procedures such as statistical modelling techniques can be used to analyse complex relationships between variables that may be related to errors and adverse events. This is particularly important given that most errors and adverse events occur as a result of multiple factors rather than one isolated cause or incident. Thus Hicks et al. (2006) and Field et al. (2004) demonstrate the use of computerized administration data in the identification of such adverse events and highlight the potential of systems for alerting impending errors through the enactment of alert signals. The addition of decision-support systems (CDSS) to computerized physician ordering systems (CPOE) further enhances the potential for patient safety (Joanna Briggs Institute 2006). Many of the suggested uses of information management technology centre on the development of electronic charting using standardized languages (Von Krogh et al. 2005). This is seen to provide a basis for decision support systems, bringing positive outcomes in relation to quality assurance, problem solving and caring (Von Krogh et al. 2005).

Improvements in clinical safety and quality of care have been linked to the need for staff development, education and training (Dunn 2003). Increasing the prominence of risk management concerns within postgraduate medical training programmes will contribute to improvements in the safety record of medical practitioners (Cowan \& Kavanagh 2005). Hospitals with the lowest mortality ratios had a comprehensive educational support system such as focused nurse education programmes and clinical nurse specialists (Knaus et al. 1986). Nurses are key frontline staff and in addition to their responsibilities for the quality of nursing care delivered they are often the medium through which other professionals and staff interface with patients. Thus, surveillance of the entire patient experience is implicit in the role of the professional nurse. Thus, the education and ongoing educational reinforcement of nurses is a key component in reducing error in health care (Dunn 2003) and should be undertaken in the context of a multidisciplinary approach. Tourangeau et al. (2006) identified four initiatives to minimize unnecessary patient death and improve patient safety (see Table 4). Understanding the multiple determinants of adverse events can lead to the development of strategies to promote safe clinical practices and outcomes 
Table 4

Initiatives to minimize unnecessary patient death and improve patient safety

Maximize the number of registered nurse within nursing staff

Develop and implement approaches that strengthen collaborative relationships among nurses and physicians

Maximize the proportion of board certified or medical specialists care providers in hospital medical staff

Establish and sustain clinical nursing support systems to enhance the delivery of patient care

Source: Tourangeau et al. (2006).

that will reduce patient mortality rates (Tourangeau et al. 2006).

\section{Conclusions}

Adverse events occur with considerable frequency in health care. They have considerable financial and human consequences for the patient, healthcare provider and society at large. Nursing professionals are uniquely placed to understand and interpret the complexity of health care. Nurse Managers are equipped to evaluate the system issues which impact on the delivery and organization of care and provide leadership to both nursing and other health professionals and staff in seeking understanding of why adverse events happen. The key to reaching effective solutions to the problem of adverse events is reaching understanding of the multiple factors that cause unsafe clinical practice situations and negative outcomes for recipients of health care. A cultural shift is necessary within health care, to achieve work environments, which support safe and open disclosure with forthright discussion of these multi-faceted events with all personnel. Only then will organizations be capable of producing clear, accessible, accurate data, which can be used to effect changes in clinical practice and promote patient safety.

\section{References}

Baker G.R., Norton P., Flintoft V. et al. (2004) The Canadian Adverse Events Study: the incidence of adverse events among hospital patients in Canada. Canadian Medical Association Journal 170 (11), 1678-1686.

Becher E.C. \& Chassin M.R. (2001) Improving quality, minimizing error: making it happen. Health Affairs 20 (3), 68-81.

Brennan T.A., Leape L.L., Laird N.M. et al. (1991) Incidence of adverse events and negligence in hospitalized patients: results of the Harvard Medical Practice Study. New England Journal of Medicine 324 (6), 324-376.

Classen D.C., Pertotnik S.L., Evans R.S., Lloyd J.F. \& Burke J.P. (1997) Adverse events in hospitalized patients: excess length of stay, extra costs, and attributable mortality. Journal of the American Medical Association 277 (4), 301-306.
Collins (1999) English Dictionary. Harper Collins Publishers, Glasgow.

Considine J. \& Botti M. (2004) Who, when and where? Identification of patients are risk of an in-hospital adverse event: implications for nursing practice. International Journal of Nursing Practice 10, 21-31.

Cowan J. \& Kavanagh S. (2005) Foundation years training and patient safety - a comprehensive introduction? Clinical Governance 10 (3), 254-260.

Davis P., Lay-Yee R., Briant R., Wasan A., Scott A. \& Schug S. (2002) Adverse incidents in New Zealand public hospitals 1: occurrence and impact. The New Zealand Medical Journal 115 (1167), 1-9.

Department of Health (1999) Clinical Governance: Quality in the New NHS. The Stationery Office, London.

Department of Health (2000) An Organisation With a Memory: Report of an Expert Group on Learning From Adverse Events in the NHS Chaired by the Chief Medical Officeri. Stationary Office, London.

Department of Health and Children (2001) Quality and Fairness - A Health System for You. Stationary Office, Dublin.

Doolan D.F. \& Bates D.W. (2002) Computerised physician order entry systems: mandates and incentives. Health Affairs 21 (4), 180-188.

Dunn D. (2003) Incident reports - correcting processes and reducing errors. Association of Operating Room Nurses 78 (2), 212-233.

Edwards I.R. \& Aronson J.K. (2000) Adverse drug reactions: definitions, diagnosis, and management. The Lancet 356, 1255-1259.

Esmail A. (2006) Clinical perspectives on patient safety. In Patient Safety: Research Into Practice (K. Walshe \& R. Boaden eds), pp. 9-18. Open University Press, Berkshire.

Field T., Gurwitz J., Harrold L. et al. (2004) Strategies for detecting adverse drug events among older persons in the ambulatory setting. Journal of the American Informatics Association 11, 492-498.

Fine A. (2004) Fatal medical errors estimated to be larger than expected. Managed Care Quarterly 12 (4), 12-13.

Gorowitz J. \& MacIntyre A. (1976) Towards a theory of Medical Fallibility. In Science, Ethics and Medicine (H.T. Engelhart \& D. Callahan eds), pp. 248-274. Hastings Centre, Hastings-onHudson, NY.

Hackman J.R. \& Wageman R. (1995) Total quality management: empirical, conceptual and practical issues. Administrative Science Quarterly 40 (2), 309-342.

Health Care Commission (2006) State of Health Care. Commission for Healthcare Audit Inspection, London.

Hiatt H. (1989) A study of medical injury and medical malpractice: an overview. New England Journal of Medicine 321, 480484.

Hicks R., Becker S. \& Cousins D. (2006) Harmful medication errors in children: a 5-year analysis of data from the USP's MEDMARK ${ }^{\circledR}$ program. Journal of Paediatric Nursing: Nursing Care of Children and Families 21 (4), 290-298.

Howard R.L. (2003) Investigation into the reasons for preventable drug related admissions to a medical admission unit: observational study. Quality and Safety in Health Care 12, 280-285.

Institute of Medicine (1999) To Err is Human: Building a Safer Health System. National Academy Press, Washington D.C. 
International Conference on Harmonisation (1996) ICH Harmonised Tripartite Guideline. Guideline for Good Clinical Practice, E6 (R1). Available at: http://www.ich.org/coche/ compo/475-272-1.html \# E6, accessed on 16 May 2008.

James T. (2004) Medical errors: the perspective of the insurer. Journal of Surgical Oncology 88, 195-199.

JCAHO (2005) Health Care at the Crossroads: Strategies for Improving the Medical Liability System and Preventing Patient Injury. Available at: http://www.jointcommission.org/ NR/rdonlyres/167DD821-A395-48FD-87F9-6AB12BCACB0F/ 0/Medical_Liability.pdf, accessed on 20 February 2007.

Joanna Briggs Institute (2006) Strategies to reduce medication errors with reference to older adults. Nursing Standard 20 (41), 53-57.

Johnson J.A., Brennan T.A, Newhouse J.P. et al. (1992) The economic consequence of medical injuries. Implications for a no-fault insurance plan. Journal of the American Medical Association 267 (18), 2487-2492.

Kable A.K., Gibberd R.W. \& Spigleman A.D. (2002) Adverse events in surgical patients in Australia. International Journal of Quality in Health Care 14 (4), 269-276.

Kalish R.L., Daley J., Duncan C.C., Davis R.B., Coffman G.A. \& Iezzoni L.I. (1995) Costs of potential complications for care for major surgery patients. American Journal of Medical Quality 10 (1), 48-54.

Karson A. \& Bates D. (1999) Screening for adverse events. Journal of Evaluation in Clinical Practice 5 (1), 23-32.

Kelley E. \& Hurst J. (2006) OECD Health Working Papers No. 22 Health Care Quality Indicators Project Initial Indicators Report. Organisation for Economic Co-operation and Development, Paris.

Knaus W.A., Draper E.A., Wagner D.P. \& Zimmerman J.E. (1986) An evaluation of outcome from intensive care in major medical centres. Annals of Internal Medicine 104 (3), 410-418.

Leape L.L. (1994) Error in medicine. Journal American Medical Association 272, 1851-1857.

Leape L.L. (2000) Institute of medicine medical error figures are not exaggerated. New England Journal of Medicine 284 (1), 95-97.

Leape L.L., Brennan T.A., Laird N. et al. (1991) The nature of adverse events in hospitalised patients results of the Harvard Medical Practice Study II. New England Journal of Medicine 324 (6), 377-384.

McDonald C.J., Weiner M. \& Hui S.L. (2000) Death due to medical errors are exaggerated in Institute of Medicine Report. Journal American Medical Association 284 (1), 93-95.

Moullin M. (2002) Delivering Excellence in Health and Social Care. Open University Press, Buckingham.

National Audit Office (2005) A Safer Place for Patient: Report by the Comptroller and Auditor General HC 456 Session 20052006. Available at: http://www.nao.org.uk/publications/ nao_reports/05-06/0506456.pdf, accessed on 20 February 2007.

National Patient Safety Agency (2004) Seven steps to patient safety: An overview guide for NHS staff. Available at: http:// www.npsa.nhs.uk/patientsafety/improvingpatientsafety/patientsafety-tools-and-guidance/1steps, accessed on 12th May 2008.
National Patient Safety Agency (2005) Building a Memory: Preventing Harm, Reducing Harm, Reducing Risks and Improving Patient Safety. The First Report of the National Reporting and Learning System and Patient Safety Observatory. Available at: http://www.npsa.nhs.uk/site/media/documents/1246_PSO_ Report_FINAL.pdf, accessed on 30 August 2007.

National Patient Safety Agency (2006) Quarterly National Reporting and Learning System Data Summery Summer 2006. Available at: http://www.npsa.nhs.uk/site/media/documents/ 1847_FINAL.APPENDIX.PSO_QTRLY.pdf, accessed on 20 February 2007.

Organisation for Economic Cooperation and Development (2007) Health Care Quality Indicators Project. Available at: http://www.oecd.org/health.hcqi, accessed on 10 September 2007.

Parker D. \& Lawton R. (2006) Psychological approaches to patient safety. In Patient Safety: Research Into Practice (K. Walshe \& R. Boaden eds), pp. 31-40. Open University Press, Maidenhead.

Parsley K. \& Corrigan P. (1999) Quality Improvement in Health Care: Putting Evidence Into Practice. Stanley Thorns Publishers, Cheltenham.

Roberts B. \& Hoch I. (2007) Malpractice litigation and medical costs in Mississippi. Health Economics 16 (8), 841-859.

Ruchlin H.S., Dubbs N.L. \& Callahan M.A. (2004) The role of leadership in instilling a culture of safety: lessons from the literature. Journal of Healthcare Management 49 (1), 47-59.

Schioler T., Lipczak H., Pedersen B.L. et al. (2001) Danish adverse event study. Ugeskrift for Laeger 163 (39), 5370-5380.

Thomas E.J., Studdart D.M., Newhouse J.P. et al. (1999) Costs of medical injuries in Utah and Colarado. Inquiry 36 (3), 255264.

Thomas E.J., Studdart D.M., Burstein H.R. et al. (2000a) Incidence and types of adverse events and negligent care in Utah and Colorado. Medical Care 38 (3), 247-249.

Thomas E.J., Studdart D.M., Runicman W. et al. (2000b) A comparison of iatrogenic injury studies in Australia and USA 1: Context, methods, case-mix, population, patients and hospital characteristics. International Journal for Quality in Health Care 12 (5), 371-378.

Tourangeau A.E., Cranley L.A. \& Jeffs L. (2006) Impact of nursing on hospital patient mortality: a focused review and related policy implications. Quality and Safety in Health Care 15, 4-8.

Vincent C., Neale G. \& Woloshynowych M. (2001) Adverse events in British Hospitals: preliminary retrospective record review. British Medical Journal 322, 517-519.

Von Krogh G., Dale C. \& Nåden D. (2005) A framework for integrating NANDA, NIC, and NOC terminology in electronic patient records. Journal of Nursing Scholarship 37 (3), 275-281.

West E. (2006) Sociological contributions to patient safety. In Patient Safety: Research Into Practice (K. Walshe \& R. Boaden eds), pp. 19-30. Open University Press, Maidenhead.

Wilson R.M., Runiman W.B., Gibberd R.W., Harrison B.T., Newby L. \& Hamilton J.D. (1995) The quality in Australian health care study. Medical Journal of Australia 163, 458-471.

World Health Organisation (2006) World Alliance for Patient Safety Forward Programme 2006-2007. WHO, Geneva. 